\title{
Erratum \\ Optimal Stratification and Allocation for the June Agricultural Survey
}

\author{
Jonathan Lisic, Hejian Sang, Zhengyuan Zhu, and Stephanie Zimmer
}

Erratum concerning the article "Optimal Stratification and Allocation for the June Agricultural Survey" by Jonathan Lisic, Hejian Sang, Zhengyuan Zhu, and Stephanie Zimmer published in Journal of Official Statistics, Volume 34, Number 1, 2018, pages 121-148 (https://doi.org/10.1515/jos-2018-0007).

This article has an error and related omission in the literature review, as well as some errors in the specification of the simulation. Neither of these errors affect any results in the paper or conclusions drawn.

The error and related omission in the literature review occur on page 122, paragraph 2 . The reference, Lavallée and Hidiroglou (1988) is incorrect and should be replaced with a reference to Hidiroglou (1986). Both papers are similar in that they provide methods to optimally stratify and allocate univariate populations into take-all, take-none, and takesome stratum under a coefficient of variation (CV) constraint. However, Lavallée and Hidiroglou (1988) improves on Hidiroglou (1986) by allowing for an arbitrary number of take-some strata. This important contribution should have been included on page 122 paragraph 2, revised below.

One major advantage that a priori and conditional allocation designs have over optimal stratified designs is that they are easy to obtain. Optimal stratified designs require an exploration of a combinatorial space to find an optimal design. This is a non-trivial problem for even small population and sample sizes. A solution to the problem of finding a univariate optimal stratified design using Neyman allocation for a fixed sample size was proposed by Dalenius and Hodges (1959). This method is commonly known as the cum $\sqrt{f}$ method (Särndal et al. 1991, Section 3.7 and Horgan 2006). Similar methods such as Hidiroglou (1986) and the multivariate extensions in Benedetti et al. (2010) and Benedetti and Piersimoni (2012) provide optimal designs under CV constraints, but are restricted to no more than three strata. These strata include a census (take-all), a sampled (take-some), and an unsampled (take-none) stratum for cut-off sampling. Lavallée and Hidiroglou (1988) introduced a univariate method that allows for an arbitrary number or take-some strata for the univariate case. These approaches are designed for highly skewed populations, exploiting the similarity of the underlying population to a geometric progression (Gunning et al. 2004). Benedetti and Piersimoni (2012) introduced a method for stratification which uses multiple administrative variables. This method, which is motivated by the Lavallée and Hidiroglou method, partitions the population into two strata, one which is sampled and one, which is a take-all stratum. 
The partitioning is determined such that the sample size is minimized for a target coefficient of variation of a response variable. In addition to allocations with goals of increasing precision, allocations also consider data collection costs and other practical constraints such as the method proposed by Valliant et al. (2014) to allocate sample in household surveys using Address-Based Sampling Frames and available commercial data.

The errors in the specification of the simulation occur in two areas. First, the sample size of the univariate homoscedastic case should of been $n=70$ instead of $n=23$. Second, on page 134 after Equation (11) $z_{i}$ was missing the $\gamma$ exponent in relation to $v_{i}$; $v_{i}$ is correctly defined as $v_{i}=\bar{z}_{i}^{\gamma}$ in the homoscedastic case and $v_{i}=z_{1, i}^{\gamma}$ in the heteroscedastic case.

\section{References}

Benedetti, R., M. Bee, and G. Espa. 2010. “A Framework for Cut-Off Sampling in Business Survey Design.” Journal of Official Statistics 26(4): 651-671.

Benedetti, R. and F. Piersimoni. 2012. "Multivariate Boundaries of a Self Representing Stratum of Large Units in Agricultural Survey Design." Survey Research Methods 6: 125-135.

Dalenius, T. and J.L.J. Hodges. 1959. “Minimum Variance Stratification.” Journal of the American Statistical Association 54: 88-101.

Gunning, P., J. Horgan, and W. Yancey. 2004. "Geometric Stratification of Accounting Data." Contaduría y Administración.

Hidiroglou, M.A. 1986. "The Construction of a Self-Representing Stratum for Large Units in Survey Design." The American Statistician 40(1): 27-31.

Horgan, J.M. 2006. "Stratification of Skewed Populations: A Review." International Statistical Review 74: 67-76.

Lavallée, P. and M. Hidiroglou. 1988. "On the Stratification of Skewed Populations." Survey Methodology 14: 33-43.

Särndal, C.-E., B. Swensson, and J. Wretman. 1991. Model Assisted Survey Sampling. Springer.

Valliant, R., F. Hubbard, S. Lee, and C. Chang. 2014. "Efficient Use of Commercial Lists in US Household Sampling." Journal of Survey Statistics and Methodology 2(2): $182-209$. 